\title{
Intravenous fasudil improves in-hospital mortality of patients with right heart failure in severe pulmonary hypertension
}

\author{
Rong Jiang ${ }^{1,5}$, Zi-Sheng $\mathrm{Ai}^{2,5}$, Xin Jiang ${ }^{3}$, Ping Yuan ${ }^{1}$, Dong Liu ${ }^{1}$, Qin-Hua Zhao ${ }^{1}$, Jing $\mathrm{He}^{1}$, Lan Wang ${ }^{1}$, \\ Mardi Gomberg-Maitland ${ }^{4}$ and Zhi-Cheng Jing ${ }^{1,3}$
}

The in-hospital mortality of severe pulmonary hypertension (PH) with right heart failure (RHF) is high despite the use of vasoactive and $\mathrm{PH}$-specific therapies. We conducted a prospective analysis evaluating the safety and outcomes of fasudil hydrochloride (Chuan Wei) therapy in acute RHF. PH patients hospitalized between April 2009 and November 2010 were treated with $30 \mathrm{mg}$ of i.v. fasudil three times daily over $30 \mathrm{~min}$, until they experienced relief of RHF symptoms. Adverse and serious adverse events were recorded. Odds ratios (ORs) and $95 \%$ confidence intervals were calculated for both in-hospital mortality and re-hospitalization. Multivariate adjustments were made for age, gender and World Health Organization functional class. There were no significant differences between the fasudil group and the control group in demographics, hemodynamics, and PH-specific and vasoactive therapies. Of the 209 study patients, 3 of the 74 patients $(4.1 \%)$ in the fasudil arm died, and 19 of the 135 patients $(14.1 \%)$ in the control arm died $(P=0.005)$. Fasudil decreased both in-hospital mortality $(0 R=0.258$ (0.074-0.903); $P=0.034$ ) and 30-day re-hospitalization ( $O R=0.200(0.059-0.681) ; P=0.010)$. Fasudil was well tolerated; one patient discontinued treatment. Intravenous fasudil may be given safely in patients with PH and acute RHF, and may reduce the rates of both in-hospital mortality and 30-day re-hospitalization.

Hypertension Research (2015) 38, 539-544; doi:10.1038/hr.2015.33; published online 19 March 2015

Keywords: fasudil; hospital mortality; pulmonary hypertension; right heart failure

\section{INTRODUCTION}

Pulmonary arterial hypertension (PAH), a progressive and fatal disease caused by a variety of disorders, is characterized by an increase in pulmonary vascular resistance (PVR), abnormal sustained pulmonary vasoconstriction and progressive structural remodeling of the pulmonary arteries, ultimately leading to right ventricular failure and death. ${ }^{1-3}$ Pulmonary hypertension $(\mathrm{PH})$ is an incurable disease characterized by sustained vasoconstriction, progressive vascular remodeling and irreversible right heart dysfunction. The in-hospital mortality of severe $\mathrm{PH}$ with acute right heart failure (RHF) is high despite the use of vasoactive and $\mathrm{PH}$-specific therapies, including i.v. dopamine, dobutamine, milrinone, i.v. or inhaled prostacyclin analogs, and/or oral endothelin receptor antagonists and phosphodiesterase-5 inhibitors, ${ }^{4,5}$ as well as other promising new drugs. ${ }^{6,7}$ During hospitalizations for other comorbid diseases, such as pneumonia, patients' World Health Organization functional class (WHO-FC) may deteriorate into WHO-FC III or WHO-FC IV, and often the event is fatal. ${ }^{8-10}$ There is a need to develop an effective therapy to improve these patients' short-term outcomes.

Fasudil hydrochloride (Chuan Wei), a small G-protein RhoA inhibitor, exerts acute pulmonary vasodilator effects in patients with severe PAH. ${ }^{11}$ Rho-kinase is activated in animal models of PAH associated with enhanced pulmonary vasoconstriction, proliferation, impaired endothelial vasodilator function and pulmonary vascular remodeling. ${ }^{11-17}$ A variety of preclinical and clinical studies have suggested that the Rho-kinase pathway is involved in vascular signaling in cardiovascular disease ${ }^{18,19}$ and $\mathrm{PH}^{20-26}$ Recently, a pilot doubleblinded, placebo-controlled study demonstrated that pulmonary hemodynamics exhibited improvement following oral treatment with an extended-release formulation of fasudil hydrochloride. ${ }^{27}$ The Rho-kinase inhibitor fasudil is currently approved in Japan and China as a treatment to prevent cerebral vasospasm in aneurysmal subarachnoid hemorrhage. Rho-kinase inhibitors are a promising new class of drugs for RHF in severe PH. Intravenous fasudil, a Rho-kinase inhibitor, is a promising

${ }^{1}$ Department of Cardio-Pulmonary Circulation, Shanghai Pulmonary Hospital, Tongji University School of Medicine, Shanghai, China; ${ }^{2}$ Department of Preventive Medicine, College of Medicine, Tongji University School of Medicine, Shanghai, China; ${ }^{3}$ State Key Lab of Cardiovascular Disease, Fu Wai Hospital, Chinese Academy of Medical Sciences and Peking Union Medical College, Beijing, China and ${ }^{4}$ Section of Cardiology, Department of Medicine, University of Chicago Medicine, Chicago, IL, USA

5 These authors contributed equally to this work.

Correspondence: Dr Z-C Jing, State Key Laboratory of Cardiovascular Disease, Fu Wai Hospital, Chinese Academy of Medical Sciences and Peking Union Medical College, No. 167 Beilishi Road, Beijing 100037, China.

E-mail: jingzhicheng@vip.163.com

Received 11 October 2014; revised 7 November 2014; accepted 24 November 2014; published online 19 March 2015 
agent for $\mathrm{PH}$ therapy. ${ }^{12,22,25,28}$ In this paper, we report the efficacy of i.v. fasudil in hospitalized patients with severe PH and RHF.

\section{METHODS}

The protocol for this prospective, open label study was approved by the Institutional Ethics Committee of Shanghai Pulmonary Hospital (approval number: K10-058) and conformed to the principles outlined in the Declaration of Helsinki. All participants who received fasudil treatment provided informed consent (children's guardians signed the consent form).

\section{Inclusion/exclusion criteria}

Patients were recruited from Shanghai Pulmonary Hospital, the largest referral center in China, which has a large number of patients diagnosed with idiopathic $\mathrm{PAH}{ }^{29}$ All patients were admitted and $\mathrm{PH}$ was diagnosed according to the Nice Classification. ${ }^{30}$ The $\mathrm{PH}$ patients either were receiving specific vasodilators prior to their hospitalization or were started on them during their incident hospitalization. All patients met the following criteria for the diagnosis of PH: (i) a mean pulmonary arterial pressure $(\mathrm{mPAP}) \geqslant 25 \mathrm{~mm} \mathrm{Hg}$ at rest as determined via right heart catheterization (RHC) or a pulmonary artery systolic pressure $>50 \mathrm{~mm} \mathrm{Hg}^{31}$ as determined via transthoracic echocardiography for patients who were too ill or unwilling to undergo RHC; (ii) classification as WHO-FC III or IV; and (iii) receiving vasoactive agents for RHF, with or without PAH-specific drugs (such as endothelin receptor antagonists, prostacyclin-analogues or phosphodiesterase-5 inhibitors).

Patients were excluded for the following reasons: classification as WHO-FC I or II; not combining the vasoactive medications (for example, dopamine or dobutamine); not admitted to the hospital and not assessed via an RHC or transthoracic echocardiography.

\section{Study design}

The investigators began using fasudil hydrochloride (Brand: Chuan Wei, Tianjin Chase Sun Pharmaceutical, No. 20, Quanfa Road, Tianjin Wuqing Development Area, China) in April 2009. Although the drug had not been approved for $\mathrm{PH}$ in China, it had been used off-label to treat patients with severe $\mathrm{PH}$ and acute RHF who exhibited either no response or a poor response to standard clinical therapies for cardiogenic shock. Acute RHF was defined as either new-onset or rapidly worsening heart failure requiring urgent inpatient therapy. A patient exhibiting evidence of volume overload or low cardiac output (CO) syndrome requiring i.v. diuretic therapy or vasopressor therapy was considered to have acute RHF. ${ }^{10}$

Between April 2009 and November 2010, 209 patients with PH were consecutively enrolled into this prospective cohort study. Eligible patients were randomly assigned to either an experimental group (fasudil group) or a control group (no-fasudil group) according to a pre-specified computer-generated scheme (2:1 ratio).The protocol was open label and not blinded.

Diuretics were used in all patients. Dobutamine and dopamine infusions, the preferred inotropic agents, were usually administered at initial doses of $2.0 \mu \mathrm{g} \mathrm{kg}^{-1}$ per minute and titrated up to doses of $3-10 \mu \mathrm{g} \mathrm{kg}^{-1}$ per minute in the presence of signs of persistently low $\mathrm{CO}$ (for example, low hypotension, altered mentation or oliguria). Milrinone was used at the usual maintenance dose of $0.25-0.75 \mu \mathrm{g} \mathrm{kg}^{-1}$ per minute. All patients received inotropic infusion and/or PAH-specific drugs (endothelin receptor antagonists, prostacyclinanalogues or phosphodiesterase- 5 inhibitors) until relief of severe RHF was achieved (for example, improvement of volume overload or low CO syndrome) and the patient either was discharged or died.

In the experimental group, fasudil infusions were initiated as an add-on to pre-existing therapy with vasoactive drugs and/or PAH-specific drugs; $30 \mathrm{mg}$ of fasudil was intravenously injected over $30 \mathrm{~min}$ three times daily until relief of acute RHF was achieved. Doses were reduced by half for children.

\section{Outcome measures}

Assessment of clinical outcomes was based on in-hospital mortality and 30-day re-hospitalization for cardiovascular symptoms. We did not observe more than one re-hospitalization for any patient enrolled in our study. Data were obtained during follow-up or via telephone interview. Any adverse events that occurred during administration of fasudil were recorded. Routine blood, liver and renal function parameters were assessed before and after the study.

\section{Hemodynamic studies}

RHC was performed in some of the patients $(n=58 / 121)$ during hospitalization. Baseline hemodynamic variables, including mPAP, mean right atrial pressure (mRAP) and pulmonary capillary wedge pressur, were measured. CO was measured in triplicate using a thermodilution technique with ice-cold isotonic sodium chloride solution, except in unrepaired congenital heart disease, in which $\mathrm{CO}$ was measured using the indirect Fick method. Cardiac index was calculated by dividing CO by body surface area. PVR) was calculated by dividing the difference between mPAP and pulmonary capillary wedge pressure by $\mathrm{CO}$.

\section{Statistical analysis}

Baseline characteristics are reported either as the mean \pm s.d. or as medians and quartiles for continuous variables, and as percentages for categorical variables. The Kolmogorov-Smirnov test was applied to assess data distribution. Baseline characteristics of study participants were compared between groups using the independent samples $t$-test, and the Mann-Whitney U-test was used to assess data that were not normally distributed. The chi-square test was used for dichotomous variables. The predefined endpoints were in-hospital mortality and re-hospitalization. Odds ratios (ORs) and 95\% confidence intervals (CIs) were calculated for in-hospital mortality and re-hospitalization.

Binary logistic regression was used to assess bivariate relationships. A two-tailed $P$ value less than 0.05 indicated a statistically significant difference.

Univariate regression analyses for in-hospital mortality and 30-day re-hospitalization were performed first. Risk factors at the 0.05 level were significant. The pre-specified risk factors were sex, age, WHO-FC, tricuspid annular plane systolic excursion, laboratory test results such as for $\mathrm{N}$-terminal pro-B type natriuretic peptide, systolic blood pressure, diastolic blood pressure, heart rate, hemodynamic variables $(\mathrm{CO}, \mathrm{mRAP}, \mathrm{mPAP}, \mathrm{PVR}$ and pulmonary capillary wedge pressure), $\mathrm{PAH}$-specific drugs (i.v./inhaled prostacyclin analogs, and/or oral endothelin receptor antagonists or phosphodiesterase-5 inhibitors) or vasoactive agents (i.v. dopamine, dobutamine and milrinone). Each continuous value was transformed into a binary variable, using either its mean or median as a cutoff.

Multivariate logistic regression analysis was performed to determine the relationship between in-hospital mortality and fasudil administration, adjusted for WHO-FC. Using multivariate logistic regression, the relationship between 30-day re-hospitalization and fasudil was analyzed, adjusting for dobutamine We reported ORs with 95\% CIs. The analyses were performed using specific statistical software (SPSS software, version 15.0, Chicago, IL, USA).

\section{RESULTS}

\section{Baseline characteristics}

A total of 209 consecutive patients with RHF-related symptoms met the inclusion criteria and were prospectively entered into this study. The study population included patients with idiopathic PAH $(37.8 \%)$, PAH associated with congenital heart disease (17.2\%), PAH associated with connective-tissue disease (13.9\%), chronic thromboembolic $\mathrm{PH}$ (15.3\%), PH with lung disease (9.0\%) and other forms of $\mathrm{PH}(7.7 \%)$ (Table 1). A total of 74 patients treated with fasudil and 135 control subjects were studied. Among the 74 patients treated with fasudil (27 male and 47 female), the median age was 42.0 (29.5-58.3) years and the mean body mass index was $21.35(14.35-30.08) \mathrm{kg} \mathrm{m}^{-2}$. The 135 control subjects ( 50 male and 85 female) had a median age of 21.35 (14.35-30.08) years and a median body mass index of 21.35 (14.35-30.08) $\mathrm{kgm}^{-2}$. Of the patients in the experimental group, 22 (29.7\%) were classified as WHO-FC IV and 52 (70.3\%) as WHO-FC III. Of the patients in the control group, $29(21.5 \%)$ were classified as WHO-FC IV and 106 (78.5\%) as WHO-FC III. The demographic and clinical characteristics of the two groups at the beginning of study are shown in Table 1. 
Table 1 Baseline characteristics of fasudil group and no-fasudil group

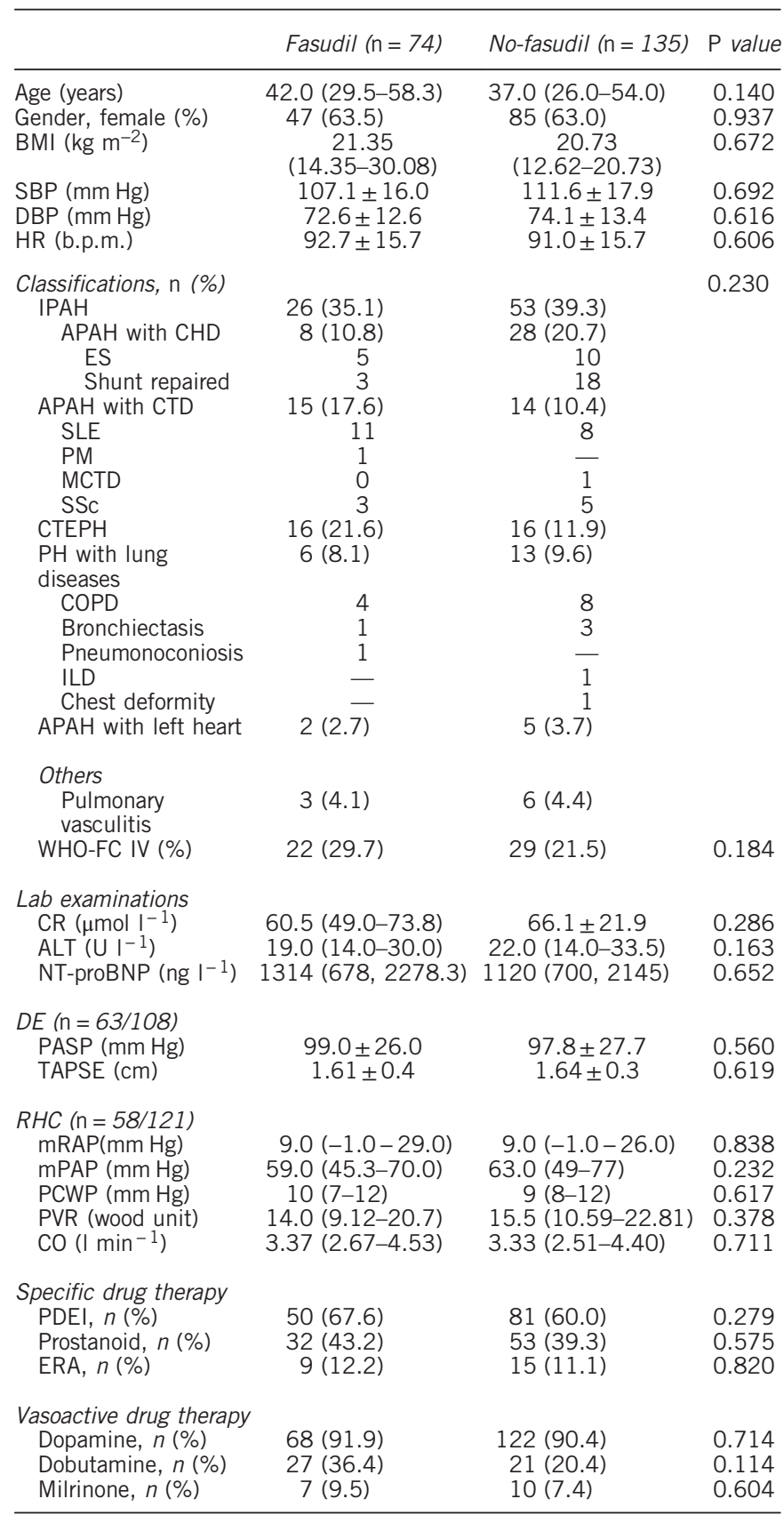

Abbreviations: ALT, alanine transaminase; APAH, associated pulmonary arterial hypertension; $\mathrm{BMI}$, body mass index; $\mathrm{CHD}$, congenital heart disease; $\mathrm{Cl}$, confidence interval; $\mathrm{CO}$, cardiac output; CR, creatinine; CTD, connective tissue disease; CTEPH, chronic thromboembolic pulmonary hypertension; COPD, chronic obstructive pulmonary diseases; DBP, diastolic blood pulmonary hypertension; COPD, chronic obstructive pulmonary diseases; DBP, diastol
pressure; DE, Doppler echocardiography; ERA, endothelin receptor antagonist; ES, Eisenmengersyndrome; HR, heart rate; ILD, interstitial lung disease; IPAH, idiopathic pulmonary artery hypertension; MCTD, mixed connective tissue disease; MPAP, mean pulmonary artery hypertension; mRAP, mean right atrium pressure; NT-proBNP, N-terminal pro-Btype natriuretic peptide; PASP, pulmonary artery systolic pressure. PCWP, pulmonary capillary wedge pressure PDEL, Phosphodiesterase type-5 inhibitors; PM, polymyositis; PVR, periphery vessel resistance; PDE, Phosphodistease type-5 inhibit erythematous; SSc, systemic sclerosis; TAPSE, tricuspid annular plane systolic excursion; WHO-FC, World Health Organization functional class.

Values are expressed as $n(\%)$ or mean \pm s.d. or median (25th, 75th percentiles).

There were no significant differences between the experimental group and the control group regarding age, sex, body mass index, classifications, WHO-FC, systolic blood pressure, diastolic blood pressure, heart rate, laboratory test results, tricuspid annular plane systolic excursion and pulmonary artery systolic pressure by echocardiogram, or hemodynamic parameters as determined via catheterization (mRAP, mPAP, pulmonary capillary wedge pressure, PVR and CO) (Table 1).

At baseline, there were no differences between the two groups regarding the use of either $\mathrm{PAH}$-specific drugs or vasoactive agents (Table 1).

\section{Outcomes}

Thirty-three patients received $>1$ week therapy; the longest duration of fasudil therapy was 28 days. The median duration of fasudil treatment was 8 days $(5,13.5)$. There were 19 deaths $(8$ males and 11 females; 8 were children and 1 was an elderly patient $\geqslant 60$ years of age) in the control group vs. 3 deaths in the fasudil group (all female patients).

\section{Logistic regression model}

The in-hospital mortality in both groups was $10.5 \%$. The in-hospital mortality was $4.1 \%$ in the fasudil group compared with $14.1 \%$ in the control group $(P=0.005)$. The unadjusted OR for both in-hospital mortality and 30-day re-hospitalization owing to RHF was 0.258 (95\% CI: $0.074-0.903 ; \quad P=0.034)$ and 0.200 (95\% CI: $0.059-0.681$; $P=0.010)$. The predictors of in-hospital mortality and 30-day rehospitalization are shown in Table 2. WHO-FC was associated with an increased risk of in-hospital mortality. Dobutamine administration was associated with an increased risk of 30-day re-hospitalization. Using multivariate logistic regression to adjust for WHO-FC, inhospital mortality was associated with fasudil administration (adjusted OR, $0.141 ; 95 \% \mathrm{CI}, 0.036-0.549 ; P=0.005$ ). The multivariate logistic regression analysis of dobutamine and fasudil administration demonstrated that only fasudil was associated with 30-day re-hospitalization (adjusted OR, 0.214; 95\% CI, 0.063-0.733; $P=0.014$ ) (Table 3).

\section{Adverse events}

One patient discontinued therapy because of an allergic rash. Of the patients treated with fasudil, none had abnormalities in hepatic or renal function during follow-up. The routine blood, liver and renal function parameters were not significantly different following treatment compared with before treatment $(P>0.05)$ (Table 4$)$

\section{DISCUSSION}

In this study, we evaluated the efficacy of i.v. fasudil in hospitalized patients with severe $\mathrm{PH}$ and RHF. Our study is the first to identify fasudil as a potential therapy for this syndrome. Fasudil improved both in-hospital mortality and 30-day re-hospitalization and was well tolerated.

Acute RHF in patients with PH is often fatal. In a recent study by Sztrymf and colleagues, ${ }^{9}$ patients with $\mathrm{PAH}$ who were hospitalized for acute heart failure in the intensive care unit had a mortality rate of $42 \% .{ }^{10}$ In our study, the overall in-hospital mortality for both groups was $10.5 \%$, lower than that reported by Sztrymf et al. ${ }^{9,10}$ In the study by Sztrymf and colleagues, all patients had severe heart failure and required hospitalization in an intensive care unit to manage low $\mathrm{CO}$ and its sequelae. However, our study included more $\mathrm{PH}$ patients classified as WHO-FC III during hospitalization.

Why did fasudil hydrochloride infusion reduce in-hospital mortality and 30-day re-hospitalization in our study? We believe that fasudil hydrochloride infusion improves hemodynamic parameters. $22,24,25,27$ Fukomoto et al., ${ }^{25}$ in 2005 , reported a statistically significant $17 \%$ reduction in PVR without a change in systemic blood pressure, although there was no significant change in either mPAP or CI. In 
Table 2 Univariate analysis for 30-day re-hospitalization and in-hospital mortality

\begin{tabular}{|c|c|c|c|c|}
\hline & \multicolumn{2}{|c|}{ 30-day re-hospitalization } & \multicolumn{2}{|c|}{ In-hospital mortality } \\
\hline & OR $(95 \% \mathrm{Cl})$ & $\mathrm{P}$ value & OR $(95 \% \mathrm{Cl})$ & $\mathrm{P}$ value \\
\hline Gender & $0.574(0.286-1.150)$ & 0.117 & $0.961(0.386-2.389)$ & 0.961 \\
\hline WHO-FC & $1.647(0.775-3.501)$ & 0.194 & $15.300(5.279-44.344)$ & $<0.001$ \\
\hline $\mathrm{SBP}(\mathrm{mm} \mathrm{Hg})$ & $0.977(0.949-1.006)$ & 0.126 & $1.004(0.901-1.118)$ & 0.946 \\
\hline Group (fasudil/no fasudil) & $0.200(0.059-0.681)$ & 0.010 & $0.258(0.074-0.903)$ & 0.034 \\
\hline $\operatorname{mRAP}(\mathrm{mm} \mathrm{Hg}),<9.0$ vs. $\geqslant 9.0$ & $1.185(0.530-2.650)$ & 0.679 & $1.015(0.336-3.066)$ & 0.979 \\
\hline mPAP $(\mathrm{mm} \mathrm{Hg}),<62.9$ vs. $\geqslant 62.9$ & $0.895(0.486-1.649)$ & 0.722 & $3.51(0.993-14.160)$ & 0.051 \\
\hline PVR (wood units), $<15.0$ vs. $\geqslant 15.0$ & $1.125(0.544-2.367)$ & 0.754 & $1.639(0.515-5.214)$ & 0.403 \\
\hline $\mathrm{CO}(1 / \mathrm{min}),<3.33 \mathrm{vs} . \geqslant 3.33$ & $0.778(0.264-1.661)$ & 0.516 & $0.673(0.205-2.205)$ & 0.513 \\
\hline $\mathrm{CR}\left(\mu \mathrm{mol} \mathrm{I} \mathrm{I}^{-1}\right)$ & $0.870(0.621-1.218)$ & 0.418 & $1.002(0.997-1.006)$ & 0.442 \\
\hline PDEI & $1.366(0.562-3.317)$ & 0.491 & $0.830(0.324-2.129)$ & 0.699 \\
\hline Prostanoid & $0.736(0.348-1.553)$ & 0.421 & $1.922(0.790-4.674)$ & 0.151 \\
\hline ERA & $1.209(0.356-4.104)$ & 0.761 & $0.650(0.140-3.021)$ & 0.583 \\
\hline Dopamine & $4.079(0.773-21.535)$ & 0.098 & $1.881(0.547-6.469)$ & 0.316 \\
\hline Dobutamine & $2.364(1.122-4.981)$ & 0.024 & $1.937(0.775-5.021)$ & 0.154 \\
\hline Milrinone & $0.941(0.110-8.631)$ & 0.981 & $1.618(0.181-14.491)$ & 0.667 \\
\hline
\end{tabular}

Abbreviations: ALT, alanine transaminase; $\mathrm{Cl}$, confidence interval; CO, cardiac output; CR, creatinine; DBP, diastolic blood pressure; DE, Doppler echocardiography; ERA, endothelin receptor antagonist; HR, heart rate; mPAP, mean pulmonary artery hypertension; mRAP, mean right atrium pressure; NT-proBNP, N-terminal pro-Btype natriuretic peptide; OR, odds ratio; PDEI, Phosphodiesterase type-5 inhibitors; PVR, periphery vessel resistance; SBP, systolic blood pressure; TAPSE, tricuspid annular plane systolic excursion; WHO-FC, World Health Organization Phosphodiesterase

functional class.

Table 3 Multivariate-adjusted OR for 30-day re-hospitalization and in-hospital mortality.

\begin{tabular}{|c|c|c|c|c|}
\hline & \multicolumn{2}{|c|}{ 30-day re-hospitalization } & \multicolumn{2}{|c|}{ In-hospital mortality } \\
\hline & OR $(95 \% \mathrm{Cl})$ & $\mathrm{P}$ value & OR $(95 \% \mathrm{Cl})$ & $\mathrm{P}$ value \\
\hline WHO-FC & - & - & $21.236(6.916-65.202)$ & $<0.001$ \\
\hline Group (fasudil/no fasudil) & $0.214(0.063-0.733)$ & 0.014 & $0.141(0.036-0.549)$ & 0.005 \\
\hline Dobutamine & $2.613(1.011-4.628)$ & 0.047 & - & - \\
\hline
\end{tabular}

Abbreviations: $\mathrm{Cl}$, confidence interval; OR, odds ratio; WHO-FC, World Health Organization functional class.

The multivariate-adjusted hazard ratios $(95 \% \mathrm{Cls})$ for risk of hospital mortality. Abbreviations as in Table 1 .

2006, Ishikura and colleagues ${ }^{22}$ described eight patients with PAH who were treated using the same fasudil regimen. There was statistically significant improvement in total peripheral resistance, mPAP and CI. The total peripheral resistance/systematic vessel resistance (SVR) ratio exhibited a decrease that was not statistically significant. In 2009, Li et al. $^{24}$ studied 12 pediatric patients scheduled for shunt closure. A dose of $30 \mathrm{mg}$ of fasudil over $30 \mathrm{~min}$ resulted in a $33 \%$ reduction in PVR, and there was a non-significant $23.9 \%$ reduction in the PVR/ SVR ratio. Fujita et al. ${ }^{23}$ found that fasudil resulted in statistically significant reductions in $\mathrm{MPAP}$ and the PVR/SVR ratio as well as in the level of nitric oxide. More recently, Fukumoto and colleagues ${ }^{27}$ described the first double-blinded, placebo-controlled trial with oral fasudil in PAH patients. The results of mid-term treatment with fasudil demonstrated an improvement in CI compared with placebo.

We did not collect hemodynamic or molecular biological data for elucidating the mechanisms responsible for the favorable effects of fasudil in our study, but we surmised that the effects on the hemodynamics and molecular biology of $\mathrm{PH}$ patients-supported by the above studies-may contribute to reductions in the rates of both in-hospital mortality and 30-day re-hospitalization.

The selectivity of fasudil for the pulmonary arterial bed suggests that it may play a role in the management of critically ill hospitalized patients with $\mathrm{PH}$. A common clinical challenge faced by clinicians involves instituting effective therapy without exacerbating systemic vasodilatation.

RAP, PAP, PVR, CO and N-terminal pro-B type natriuretic peptide are important prognostic factors of mortality in $\mathrm{PAH}$; a predictive relationship between these parameters and mortality has been reproduced in many studies. ${ }^{32-35}$ However, in our study, RAP, PAP, PVR and CO were not associated with in-hospital mortality $(P>0.05)$. We believe that there are two reasons for this finding. First, in-hospital mortality was a short-term prognosis rather than a long-term prognosis. The observation time may have been too short to influence mortality in patients with acute RHF and $\mathrm{PH}$ patients classified as 
Table 4 The safety of i.v. fasudil hydrochloride

\begin{tabular}{lccr}
\hline & Baseline & After treatment & P value \\
\hline RBC $\left(\times 10^{12} \mathrm{I}^{-1}\right)(n=43)$ & $4.82 \pm 0.93$ & $4.67 \pm 0.73$ & 0.121 \\
WBC $\left(\times 10^{9} \mathrm{I}^{-1}\right)(n=43)$ & $6.25(5.23,8.40)$ & $7.20(5.50,9.30)$ & 0.170 \\
PLT $\left(\times 10^{9} \mathrm{I}^{-1}\right)(n=43)$ & $160.14 \pm 63.83$ & $177.86 \pm 78.85$ & 0.051 \\
ALT $\left(\mathrm{IU} \mathrm{I} \mathrm{I}^{-1}\right)(n=49)$ & $18.50(14.00,30.00)$ & $22.00(15.00,33.00)$ & 0.083 \\
AST $\left(\mathrm{IU} \mathrm{I} \mathrm{I}^{-1}\right)(n=49)$ & $26.00(20.00,34.25)$ & $28.39 \pm 9.73$ & 0.917 \\
$\mathrm{Cr}\left(\mu \mathrm{mol} \mathrm{I}^{-1}\right)(n=45)$ & $60.00(48.75,73.00)$ & $65.00(52.00,77.00)$ & 0.491 \\
BUN $\left(\mathrm{mmol} \mathrm{I}^{-1}\right)(n=45)$ & $6.17 \pm 2.21$ & $6.20(5.35,8.00)$ & 0.445 \\
\hline
\end{tabular}

Abbreviations: ALT, alanine aminotransferase; AST, aspartate transaminase; BUN, blood urea nitrogen; $\mathrm{Cr}$, creatinine; PLT, blood platelet.

Values are expressed as $n(\%)$ or mean \pm s.d. or median (25th, 75th percentiles).

WHO-FC III or WHO-FC IV; RAP, PAP, PVR and CO may have diminished the impact of the other parameters on patients' short-term prognoses.

The second reason stems from the fact that dobutamine plays a more important role in RV contractility than dopamine. ${ }^{36-40}$ In our center, only $20.4-36.4 \%$ of the patients were treated with dobutamine; most of the patients received dopamine (Table 1) owing to low blood pressure. We did not have enough previous experience regarding this issue. Now, however, dobutamine is the first line of inotropic therapy for $\mathrm{PH}$ patients in our center.

\section{Study limitations}

This study did not standardize the background treatment approaches to acute RHF; therefore, there may have been better or poor care provided to the groups. Additionally, differences in time collection may have introduced the following confounders: (i) patient severity bias, (ii) improved diagnostic techniques and treatments during a more recent treatment period and (iii) differences in data integrity and follow-up between different treatment periods. Some of the patients did not undergo RHC in this study. Of these, 15 patients with $\mathrm{PH}$ had undergone an RHC several years previously at our center and did not undergo a repeat procedure during this study. Because it was not appropriate to use their hemodynamic parameters to analyze their risk factors for disease mortality, we did not analyze the hemodynamic parameters of these patients. Three patients were diagnosed via an RHC after recovering and returning for a follow-up visit. Patients who did not undergo an RHC were diagnosed with $\mathrm{PH}$ according to their symptoms, signs, history suggestive of $\mathrm{PH}$ and echocardiographic findings. This was a limitation of our study. However, the focus of our study was severe RHF as opposed to PAH. Therefore, the lack of RHC data was acceptable because it is difficult for $\mathrm{PH}$ patients with severe RHF to undergo an RHC. We will devote more attention to this problem in future studies.

Our study was a short-term study rather than a long-term study. Medication administered during hospitalization will only affect a patient's short-term prognosis; it cannot influence the long-term outcome, which may be affected by many factors. Therefore, we did not include follow-up hemodynamic and echocardiographic data in our study. The primary clinical outcomes in our study were inhospital mortality and 30-day re-hospitalization for cardiovascular symptoms. Information regarding re-hospitalization was obtained either during follow-up or via telephone interview. We did not assess secondary outcome measures such as serum markers or echocardiography, and we recognize that this may represent another limitation of our study. We will design more comprehensive studies in the future.

\section{CONCLUSION}

Rho-kinase inhibitors are a promising new class of drugs for the treatment of $\mathrm{PH}$ and acute RHF. Intravenous fasudil appears to be safe and may reduce the rates of both in-hospital mortality and rehospitalization among these patients. This report represents another step toward even more effective treatments for a deadly disease. However, a prospective trial, with a standardized background protocol, is needed.

\section{CONFLICT OF INTEREST}

Dr Z-C Jing serves as a consultant and scientific advisor to Actelion, Bayer Schering, AstraZeneca, Pfizer and United Therapeutics, in addition to being an investigator in trials sponsored by these companies. Actelion, Gilead, Medtronic, Novartis, Lung Biotechnology, Reata, and Ventripoint have provided funding to the University of Chicago to support Dr Gomberg-Maitland's conduct of clinical trials. Dr Gomberg-Maitland has served as a consultant for Actelion, Bayer, Gilead, Medtronic, Bellerophon (formerly known as Ikaria) and United Therapeutics, as a member of steering committees and DSMB/event committees. She has received honoraria for CME from Medscape and ABComm. Dr Gomberg-Maitland is a member of the PCORI Advisory Panel on Rare Diseases and a Special Government Employee in the FDA's CardioRenal Division. The other authos declare no conflict of interest.

\section{ACKNOWLEDGEMENTS}

This study was partially supported by the Program for Shanghai's Outstanding Academic Leaders, the 'Dawn' Program of the Shanghai Education

Commission (10SG25), the Project of Shanghai Pulmonary Hospital (fk1011) and the Special Scientific Research Fund Project for the 'Peking Union Scholars Special Term Professor.' The sponsors had no involvement in the study design, data analysis, data interpretation or writing or revision of the report.

1 Rubin LJ. Primary pulmonary hypertension. N Engl J Med 1997; 336: 111-117.

2 Rabinovitch M. Pulmonary hypertension: updating a mysterious disease. Cardiovasc Res 1997; 34: 268-272.

3 Humbert M, Sitbon O, Simonneau G. Treatment of pulmonary arterial hypertension. N Engl J Med 2004; 351: 1425-1436.

4 Hirashiki A, Kondo T, Murohara T. Combination therapy adding tadalafil to existing ambrisentan in patients with pulmonary arterial hypertension. Hypertens Res 2014; 37: 488-489.

5 Zhuang Y, Jiang B, Gao H, Zhao W. Randomized study of adding tadalafil to existing ambrisentan in pulmonary arterial hypertension. Hypertens Res 2014; 37: 507-512.

6 Schermuly RT, Janssen W, Weissmann N, Stasch JP, Grimminger F, Ghofrani HA. Riociguat for the treatment of pulmonary hypertension. Expert Opin Investig Drugs 2011; 20: 567-576

7 Guo Q, Huang JA, Yamamura A, Yamamura H, Zimnicka AM, Fernandez R, Yuan JX. Inhibition of the $\mathrm{Ca}(2+)$-sensing receptor rescues pulmonary hypertension in rats and mice. Hypertens Res 2014; 37: 116-124.

8 Campo A, Mathai SC, Le Pavec J, Zaiman AL, Hummers LK, Boyce D, Housten T, Lechtzin N, Chami H, Girgis RE, Hassoun PM. Outcomes of hospitalisation for right heart failure in pulmonary arterial hypertension. Eur Respir J 2011; 38: 359-367.

9 Sztrymf B, Souza R, Bertoletti L, Jais X, Sitbon O, Price LC, Simonneau G, Humbert M. Prognostic factors of acute heart failure in patients with pulmonary arterial hypertension. Eur Respir J 2010; 35: 1286-1293.

10 Haddad F, Peterson T, Fuh E, Kudelko KT, de Jesus PV, Skhiri M, Vagelos R, Schnittger I, Denault AY, Rosenthal DN, Doyle RL, Zamanian RT. Characteristics and outcome after hospitalization for acute right heart failure in patients with pulmonary arterial hypertension. Circ Heart Fail 2011; 4: 692-699.

11 Fukumoto Y, Shimokawa H. [The role of Rho-kinase pathway on PAH]. Nihon Rinsho 2008; 66: 2091-2096.

12 Fukumoto $\mathrm{Y}$, Tawara S, Shimokawa $\mathrm{H}$. Recent progress in the treatment of pulmonary arterial hypertension: expectation for rho-kinase inhibitors. Tohoku J Exp Med 2007; 211: 309-320.

13 Takemoto M, Sun J, Hiroki J, Shimokawa H, Liao JK. Rho-kinase mediates hypoxiainduced downregulation of endothelial nitric oxide synthase. Circulation 2002; 106: 57-62.

14 Hyvelin JM, Howell K, Nichol A, Costello CM, Preston RJ, McLoughlin P. Inhibition of Rho-kinase attenuates hypoxia-induced angiogenesis in the pulmonary circulation. Circ Res 2005; 97: 185-191.

15 Ward JP, McMurtry IF. Mechanisms of hypoxic pulmonary vasoconstriction and their roles in pulmonary hypertension: new findings for an old problem. Curr Opin Pharmacol 2009; 9: 287-296. 
$16 \mathrm{Li} \mathrm{FH}$, Xia W, Li AW, Zhao CF, Sun RP. Inhibition of rho kinase attenuates high flow induced pulmonary hypertension in rats. Chin Med J (Engl) 2007; 120 22-29.

17 Li F, Xia W, Li A, Zhao C, Sun R. Long-term inhibition of Rho kinase with fasudil attenuates high flow induced pulmonary artery remodeling in rats. Pharmacol Res 2007; 55: 64-71

18 Nohria A, Grunert ME, Rikitake Y, Noma K, Prsic A, Ganz P, Liao JK, Creager MA. Rho kinase inhibition improves endothelial function in human subjects with coronary artery disease. Circ Res 2006; 99: 1426-1432.

19 Hirooka Y, Shimokawa $\mathrm{H}$. Therapeutic potential of rho-kinase inhibitors in cardiovascular diseases. Am J Cardiovasc Drugs 2005; 5: 31-39.

20 Abe K, Shimokawa H, Morikawa K, Uwatoku T, Oi K, Matsumoto Y, Hattori T, Nakashima Y, Kaibuchi K, Sueishi K, Takeshit A. Long-term treatment with a Rhokinase inhibitor improves monocrotaline-induced fatal pulmonary hypertension in rats. Circ Res 2004; 94: 385-393.

21 Fagan KA, Oka M, Bauer NR, Gebb SA, Ivy DD, Morris KG, McMurtry IF. Attenuation of acute hypoxic pulmonary vasoconstriction and hypoxic pulmonary hypertension in mice by inhibition of Rho-kinase. Am J Physiol Lung Cell Mol Physiol 2004; 287: L656-L664.

22 Ishikura K, Yamada N, Ito M, Ota S, Nakamura M, Isaka N, Nakano T. Beneficial acute effects of rho-kinase inhibitor in patients with pulmonary arterial hypertension. Circ J 2006; 70: 174-178.

23 Fujita H, Fukumoto Y, Saji K, Sugimura K, Demachi J, Nawata J, Shimokawa H. Acute vasodilator effects of inhaled fasudil, a specific Rho-kinase inhibitor, in patients with pulmonary arterial hypertension. Heart Vessels 2010; 25: 144-149.

24 Li F, Xia W, Yuan S, Sun R. Acute inhibition of Rho-kinase attenuates pulmonary hypertension in patients with congenital heart disease. Pediatr Cardiol 2009; 30: 363-366.

25 Fukumoto Y, Matoba T, Ito A, Tanaka H, Kishi T, Hayashidani S, Abe K, Takeshita A Shimokawa $\mathrm{H}$. Acute vasodilator effects of a Rho-kinase inhibitor, fasudil, in patients with severe pulmonary hypertension. Heart 2005; 91: 391-392.

26 Do EZ, Fukumoto Y, Takaki A, Tawara S, Ohashi J, Nakano M, Tada T, Saji K, Sugimura K, Fujita H, Hoshikawa Y, Nawata J, Kondo T, Shimokawa H. Evidence for Rho-kinase activation in patients with pulmonary arterial hypertension. Circ $J$ 2009; 73: 1731-1739.

27 Fukumoto Y, Yamada N, Matsubara H, Mizoguchi M, Uchino K, Yao A, Kihara Y, Kawano M, Watanabe H, Takeda Y, Adachi T, Osanai S, Tanabe N, Inoue T, Kubo A, Ota Y, Fukuda K, Nakano T, Shimokawa H. Double-blind, placebo-controlled clinical trial with a rho-kinase inhibitor in pulmonary arterial hypertension. Circ J 2013; 77: 2619-2625.
28 Jiang BH, Tawara S, Abe K, Takaki A, Fukumoto Y, Shimokawa H. Acute vasodilator effect of fasudil, a Rho-kinase inhibitor, in monocrotaline-induced pulmonary hypertension in rats. J Cardiovasc Pharmacol 2007; 49: 85-89.

29 Zhang R, Dai LZ, Xie WP, Yu ZX, Wu BX, Pan L, Yuan P, Jiang X, He J, Humbert M, Jing ZC. Survival of Chinese patients with pulmonary arterial hypertension in the modern treatment era. Chest 2011; 140: 301-309.

30 Hoeper MM, Bogaard HJ, Condliffe R, Frantz R, Khanna D, Kurzyna M, Langleben D, Manes A, Satoh T, Torres F, Wilkins MR, Badesch DB. Definitions and diagnosis of pulmonary hypertension. J Am Coll Cardiol 2013; 62: D42-D50.

31 Galie N, Hoeper MM, Humbert M, Torbicki A, Vachiery JL, Barbera JA, Beghetti M, Corris P, Gaine S, Gibbs JS, Gomez-Sanchez MA, Jondeau G, Klepetko W, Opitz C Peacock A, Rubin L, Zellweger M, Simonneau G. Guidelines for the diagnosis and treatment of pulmonary hypertension: the Task Force for the Diagnosis and Treatment of Pulmonary Hypertension of the European Society of Cardiology (ESC) and the European Respiratory Society (ERS), endorsed by the International Society of Heart and Lung Transplantation (ISHLT). Eur Heart J 2009; 30: 2493-2537.

32 Fijalkowska A, Kurzyna M, Torbicki A, Szewczyk G, Florczyk M, Pruszczyk P, Szturmowicz M. Serum N-terminal brain natriuretic peptide as a prognostic parameter in patients with pulmonary hypertension. Chest 2006; 129: 1313-1321.

33 Nagaya N, Nishikimi T, Uematsu M, Satoh T, Kyotani S, Sakamaki F, Kakishita M, Fukushima K, Okano Y, Nakanishi N, Miyatake K, Kangawa K. Plasma brain natriuretic peptide as a prognostic indicator in patients with primary pulmonary hypertension. Circulation 2000; 102: 865-870.

34 Chapman PJ, Bateman ED, Benatar SR. Prognostic and therapeutic considerations in clinical primary pulmonary hypertension. Respir Med 1990; 84: 489-494.

35 Barst RJ, Gibbs JS, Ghofrani HA, Hoeper MM, McLaughlin VV, Rubin LJ, Sitbon O, Tapson VF, Galie N. Updated evidence-based treatment algorithm in pulmonary arterial hypertension. J Am Coll Cardiol 2009; 54: S78-S84.

36 Hollenberg SM. Vasoactive drugs in circulatory shock. Am J Respir Crit Care Med 2011 183: 847-855.

37 Holloway EL, Polumbo RA, Harrison DC. Acute circulatory effects of dopamine in patients with pulmonary hypertension. Br Heart J 1975; 37: 482-485.

38 Kerbaul F, Rondelet B, Motte S, Fesler P, Hubloue I, Ewalenko P, Naeije R, Brimioulle $\mathrm{S}$. Effects of norepinephrine and dobutamine on pressure load-induced right ventricular failure. Crit Care Med 2004; 32: 1035-1040.

39 Chen EP, Bittner HB, Davis RJ, Van Trigt PR. Milrinone improves pulmonary hemodynamics and right ventricular function in chronic pulmonary hypertension. Ann Thorac Surg 1997; 63: 814-821.

40 Poor HD, Ventetuolo CE. Pulmonary hypertension in the intensive care unit. Prog Cardiovasc Dis 2012; 55: 187-198. 